Bull. Korean Math. Soc. 50 (2013), No. 2, pp. 475-483

http://dx.doi.org/10.4134/BKMS.2013.50.2.475

\title{
LOCALIZATION OF INJECTIVE MODULES OVER $w$-NOETHERIAN RINGS
}

\author{
Hwankoo Kim and Fanggui Wang
}

\begin{abstract}
We give some characterizations of injective modules over $w$ Noetherian rings. It is also shown that each localization of a GV-torsionfree injective module over a $w$-Noetherian ring is injective.
\end{abstract}

\section{Introduction}

One of bad properties of injective modules is that they do not preserve localization in general. However, there are some positive results as follows. If a (commutative) ring $R$ is Noetherian or hereditary, then localizations of injective $R$-modules are also injective ([10, Theorem 4.88] or [1, Proposition 2]). Recently Couchot investigated localizations of injective modules over valuation rings ([1]) and arithmetical rings ([2]). The purpose of this article is to provide another positive result if $R$ is a $w$-Noetherian ring.

We first introduce some definitions and notation from $[12,16]$. Throughout we let $R$ be a commutative ring with identity. For an $R$-module $M$, the dual module $\operatorname{Hom}_{R}(M, R)$ of $M$ is denoted by $M^{\mathrm{b}}$. Following [16, Definition 1.1], an ideal $J$ of a commutative ring $R$ is called a Glaz-Vasconcelos ideal or a $G V$-ideal, denoted by $J \in \mathrm{GV}(R)$, if $J$ is finitely generated and the natural homomorphism $\phi: R \rightarrow J^{\mathrm{b}}(\phi(r)(a)=r a$ for all $r \in R$ and $a \in J)$ is an isomorphism. Recall from [16, Definition 1.3] that an $R$-module $M$ is called a $G V$-torsion-free module if whenever $J x=0$ for some $J \in \mathrm{GV}(R)$ and $x \in M$, then $x=0$. Then it is clear that $R$ is a GV-torsion-free $R$-module, and that every submodule of a GV-torsion-free module is GV-torsion-free. It is also introduced in [16, Definition 2.1] that a GV-torsion-free $R$-module $M$ is said to be a $w$-module if, for any $J \in \mathrm{GV}(R)$, $\operatorname{Ext}_{R}^{1}(R / J, M)=0$. Then it is clear that $R$ is a $w$-module, and that for a GV-torsion-free $R$-module $M, E(M)$, the injective envelope of $M$, is a $w$-module. Note also that the concept of $w$-modules over commutative rings generalizes that of $w$-modules over integral

Received October 23, 2011; Revised January 17, 2012.

2010 Mathematics Subject Classification. Primary 13F05; Secondary 13A15, 13C13.

Key words and phrases. injective module, localization, $w$-Noetherian ring.

This research was supported by the Academic Research fund of Hoseo University in 2011(2011-0249) 
domains in $[7,13]$. Let $w$-Max $(R)$ denote the set of $w$-ideals of $R$ maximal among proper $w$-ideals of $R$ and we call $\mathfrak{m} \in w$-Max $(R)$ a maximal $w$-ideal of $R$. Then by [16, Proposition 3.8] every maximal $w$-ideal is prime. Let $M$ be a GV-torsion-free $R$-module. Then the $w$-envelope of $M$ is defined by

$$
M_{w}=\{x \in E(M) \mid J x \subseteq M \text { for some } J \in \mathrm{GV}(R)\} .
$$

It follows from [16, Theorem 2.2] that a GV-torsion-free module $M$ is a $w$ module if and only if $M_{w}=M$. So $M$ is a $w$-ideal when $M$ is an ideal of $R$ with $M_{w}=M$. We say that a GV-torsion-free module $M$ is said to be of finite type if $M_{w}=N_{w}$ for some finitely generated submodule $N$ of $M$. In [15] Wang and Zhang generalized the notion of $w$-Noetherian modules over commutative rings: A $w$-module $M$ is called a $w$-Noetherian module if $M$ has the ascending chain condition on $w$-submodules of $M$ and $R$ is said to be $w$-Noetherian if $R$ itself is a $w$-Noetherian module. It was shown in [16] that a $w$-module $M$ is $w$-Noetherian if and only if every submodule of $M$ is of finite type. If $R$ is an integral domain, then the notion of $w$-Noetherian rings is the same as that of SM (strong Mori) domains introduced by Wang and McCasland in [14]. It is shown in [16, Corollary 4.4] that if $R$ is a $w$-Noetherian ring, then $R_{\mathfrak{p}}$ is Noetherian for each prime $w$-ideal $\mathfrak{p}$ of $R$. Any undefined terminology is standard, as in $[4,5,10]$.

\section{Main result}

We begin with this section by giving a Baer-like characterization for a $w$ module.

Theorem 2.1. Let $R$ be a commutative ring and let $E$ be a w-module over $R$. Then the following statements are equivalent:

(1) $E$ is an injective $R$-module;

(2) $\operatorname{Ext}_{R}^{1}(R / I, E)=0$ for any $w$-ideal $I$ of $R$;

(3) for any $w$-ideal $I$ of $R$, a homomorphism $f: I \rightarrow E$ can be extended to $R$;

(4) for any $w$-submodule $A$ of a w-module $B$, a homomorphism $f: A \rightarrow E$ can be extended to $B$;

(5) for any $w$-submodule $A$ of a $w$-module $B$, $\operatorname{Ext}_{R}^{1}(B / A, E)=0$;

(6) for any $G V$-torsion-free module $C$, $\operatorname{Ext}_{R}^{1}(C, E)=0$.

Proof. $(1) \Rightarrow(2)$ This is trivial.

$(2) \Rightarrow(1)$ Let $I$ be an ideal of $R$ and let $f: I \rightarrow E$ be a homomorphism. Since $E$ is a $w$-module, $\operatorname{Ext}_{R}^{1}\left(I_{w} / I, E\right)=0$ by [16, Theorem 3.6]. From the exact sequence $0 \rightarrow I_{w} / I \rightarrow R / I \rightarrow R / I_{w} \rightarrow 0$, we have the exact sequence

$$
0=\operatorname{Ext}_{R}^{1}\left(R / I_{w}, E\right) \rightarrow \operatorname{Ext}_{R}^{1}(R / I, E) \rightarrow \operatorname{Ext}_{R}^{1}\left(I_{w} / I, E\right)=0 .
$$

Thus $\operatorname{Ext}_{R}^{1}(R / I, E)=0$, and therefore, $E$ is injective.

$(2) \Leftrightarrow(3)$ This is clear. 
The proofs of the other equivalences are easy or similar to those of $(1) \Leftrightarrow$ $(2) \Leftrightarrow(3)$.

Corollary 2.2. Let $E$ be a w-module. If $E$ is not injective, then there is a $w$-ideal $I$ of $R$ and a homomorphism $f: I \rightarrow E$ such that $f$ cannot be extended to $R$.

Let $R$ be an integral domain. Recall that an $R$-module $M$ is said to be divisible if for every nonunit $r \in R$ and for every $m \in M$, the equation $r x=m$ admits a solution $x \in M$.

Lemma 2.3 ([4, Lemma I.7.2]). For a module $M$ over a domain $R$ the following are equivalent:

(1) $M$ is divisible;

(2) for every $r \in R$, every homomorphism $r R \rightarrow M$ can be extended to a homomorphism $R \rightarrow M$;

(3) $\operatorname{Ext}_{R}^{1}(R / r R, M)=0$ for every $r \in R$;

(4) $\operatorname{Ext}_{R}^{1}(R / I, M)=0$ for every invertible ideal $I$ of $R$.

It is well known that a domain $R$ is a Dedekind domain if and only if every divisible $R$-module is injective, and that for a torsion-free module $M$ over an integral domain, $M$ is divisible if and only if $M$ is injective. It is also well known that an integral domain $R$ is a unique factorization domain if and only if every $w$-ideal of $R$ is principal (cf., [6]). Thus we have the following:

Corollary 2.4. Let $R$ be a unique factorization domain and let $E$ be a wmodule. Then $E$ is injective if and only if $E$ is divisible.

To give a Cohen-type theorem of Baer's criterion for GV-torsion-free injective modules over $w$-Noetherian rings, we need the following:

Lemma 2.5. Let $A$ be a w-submodule of the w-module $M$. Then for any $x \in M \backslash A$ and $r \in R, P_{r x}:=\left\{r^{\prime} \in R \mid r^{\prime}(r x) \in A\right\}$ is a $w$-ideal of $R$.

Proof. For any $r^{\prime} \in\left(P_{r x}\right)_{w}$, there exists a $J \in \mathrm{GV}(R)$ such that $J r^{\prime} \subseteq P_{r x}$. Then $J r^{\prime}(r x) \subseteq A$. Since $A$ is a $w$-module, we have $r^{\prime}(r x) \in A$. So $r^{\prime} \in P_{r x}$, and hence $P_{r x}$ is a $w$-ideal of $R$.

Theorem 2.6. Let $R$ be a w-Noetherian ring and let $A$ be a w-submodule of the $w$-module $M$. Then for any $x \in M \backslash A$, there exists $r \in R$ such that $P_{r x}=\left\{r^{\prime} \in R \mid r^{\prime}(r x) \in A\right\}$ is a prime $w$-ideal of $R$.

Proof. Let $\mathscr{C}_{x}:=\left\{P_{r x} \mid r x \notin A\right\}$. Then $P_{1 x} \in \mathscr{C}_{x}$, so $\mathscr{C}_{x}$ is nonempty. By Lemma 2.5, $P_{r x}$ is a $w$-ideal of $R$ for any $r \in R$. Since $R$ is a $w$-Noetherian ring, there exists an $r \in R$ such that $P_{r x}$ is maximal in $\mathscr{C}_{x}$. Since $P_{r x} \in \mathscr{C}_{x}$, $r x \notin A$, therefore $1 \notin P_{r x}$, that is, $P_{r x} \neq R$. For any st $\in P_{r x}$ with $s \notin P_{r x}$, we have $\operatorname{sr} x \notin A$. If $y \in P_{r x}$, then $y r x \in A$, and so $y s r x=s(y r x) \in A$. Hence $P_{s r x}=P_{r x}$. But $t(s r x)=s t(r x) \in A$, we get $t \in P_{s r x}=P_{r x}$. Therefore, $P_{r x}$ is a prime $w$-ideal of $R$. 
Theorem 2.7. Let $R$ be a $w$-Noetherian ring and let $E$ be a $w$-module over $R$. Then the following statements are equivalent:

(1) E is injective;

(2) for any prime $w$-ideal $\mathfrak{p}$ of $R$, every $R$-module homomorphism $f: \mathfrak{p} \rightarrow$ $E$ can be extended to $R$

(3) for any prime $w$-ideal $\mathfrak{p}$ of $R$, $\operatorname{Ext}_{R}^{1}(R / \mathfrak{p}, E)=0$.

Proof. (1) $\Rightarrow(2)$ By Baer's criterion.

$(2) \Rightarrow(1)$ Let $A$ be a $w$-submodule of a $w$-module $B$. Consider the following diagram:

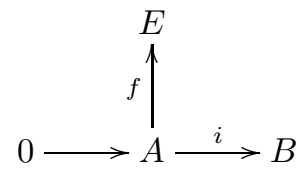

Let $\mathscr{C}$ consist of all pairs $\left(A^{\prime}, g^{\prime}\right)$, where $A \subseteq A^{\prime} \subseteq B, A^{\prime}$ is a $w$-submodule of $B$, and $g^{\prime}: A^{\prime} \rightarrow E$ extends $f$. Note that $\mathscr{C} \neq \emptyset$ for $(A, f) \in \mathscr{C}$. Define a partial order on $\mathscr{C}$ by

$$
\left(A_{1}, g_{1}\right) \leq\left(A_{2}, g_{2}\right) \text { if and only if } A_{1} \subseteq A_{2} \text { and } g_{2} \text { extends } g_{1} \text {. }
$$

By Zorn's Lemma, there exists a maximal pair $\left(A_{0}, g_{0}\right)$ in $\mathscr{C}$. If $A_{0}=B$, then we are done. Now assume that $A_{0} \neq B$. Then by Theorem 2.6, there exists an $x \in B \backslash A_{0}$ such that $P_{x}$ is a prime $w$-ideal of $R$. Define $h: P_{x} \rightarrow E$ by $h(r)=g_{0}(r x)$ for $r \in P_{x}$. By hypothesis, $h$ can be extended to $R$. Define $g: A_{0}+R x \rightarrow E$ by $g(a+r x)=g_{0}(a)+h(r), a \in A_{0}$ and $r \in R$. It is routine to verify that $g$ is well-defined. Obviously, $g$ extends $g_{0}$. So $\left(A_{0}+R x, g\right) \in \mathscr{C}$, a contradiction. Therefore $E$ is an injective module.

$(2) \Leftrightarrow(3)$ It is straightforward from the following exact sequence: $0 \rightarrow$ $\operatorname{Hom}_{R}(R / \mathfrak{p}, E) \rightarrow \operatorname{Hom}_{R}(R, E) \rightarrow \operatorname{Hom}_{R}(\mathfrak{p}, E) \rightarrow \operatorname{Ext}_{R}^{1}(R / \mathfrak{p}, E) \rightarrow 0$.

In order to give another proof of Theorem 2.7, which is suggested by the referee, we first introduce notation we need.

Let $M$ be a module and $N$ a submodule of $M$. Set $N_{(w, M)}=\{x \in M \mid$ $J x \in N$ for some $J \in G V(R)\}$. If $N_{(w, M)}=N$, then $N$ is called a relative $w$ submodule of $M$. It is clear that if $N$ is a relative $w$-submodule of $M$, then $M / N$ is $\mathrm{GV}$-torsion-free, and if $N_{(w, M)}=M$, then $M / N$ is $\mathrm{GV}$-torsion. Moreover, a relative $w$-submodule of $M$ is not a $w$-module in general, for example, the (total) $G V$-torsion submodule of a module $M$ is a relative $w$-submodule, but is not a $w$-submodule. When $M$ is a $w$-module, the relative $w$-submodules of $M$ are actually $w$-submodules of $M$.

Lemma 2.8. Let $M$ be a finite type module. Then there is a finitely generated submodule $N$ such that $N_{(w, M)}=M$.

Proof. Since $M$ is of finite type, there is a finitely generated submodule $N$ of $M$ such that $M / N$ is $\mathrm{GV}$-torsion. It is clear that $N_{(w, M)}=M$. 
The following result can be easily obtained.

Theorem 2.9. Let $M$ be a module. Then $M$ is a $w$-Noetherian module if and only if $M$ has $A C C$ on relative $w$-submodules of $M$.

Let $M$ be an $R$-module. A prime ideal $\mathfrak{p}$ of $R$ is called an associated prime ideal of $M$ if there exists $x \in M \backslash\{0\}$ such that $\mathfrak{p}$ is a prime ideal minimal over $\operatorname{ann}(x)$. As in the domain case, we have that a commutative ring $R$ is a $w$-Noetherian ring if and only if every finite type $w$-module is a $w$-Noetherian module ([15]). We can rewrite [15, Theorem 3.18] as follows:

Theorem 2.10. Let $R$ be a $w$-Noetherian ring and let $M$ be a nonzero finite type GV-torsion-free module. Then there is a finite ascending chain of relative $w$-submodules of $M$ :

$$
0=N_{0} \subset\left(N_{1}\right)_{(w, M)} \subset\left(N_{2}\right)_{(w, M)} \subset \cdots \subset\left(N_{n-1}\right)_{(w, M)} \subset\left(N_{n}\right)_{(w, M)}=M
$$

such that $\left(N_{i-1}\right)_{(w, M)} \subset N_{i}$ and $N_{i} /\left(N_{i-1}\right)_{(w, M)} \cong R / \mathfrak{p}_{i}$ for some prime $w$ ideal $\mathfrak{p}_{i}$ of $R, i=1, \ldots, n$.

Proof. Choose an associated prime ideal $\mathfrak{p}_{1}$ of $M$. Then $\mathfrak{p}_{1}$ is a prime $w$-ideal of $R$ and $\mathfrak{p}_{1}=\operatorname{ann}\left(x_{1}\right)$ for some $x_{1} \in M$. Set $N_{1}:=R x_{1} \subseteq M$. Then $N_{1}=R / \mathfrak{p}_{1}$. If $\left(N_{1}\right)_{(w, M)}=M$, then we have $0=N_{0} \subset\left(N_{1}\right)_{(w, M)}=M$, as desired. If $\left(N_{1}\right)_{(w, M)} \neq M$, then $M /\left(N_{1}\right)_{(w, M)}$ is a nonzero finite type GV-torsion-free $R$-module. Take $x_{2} \in M \backslash\left(N_{1}\right)_{(w, M)}$ such that $\left(\left(N_{1}\right)_{(w, M)}+\right.$ $\left.R x_{2}\right) /\left(N_{1}\right)_{(w, M)} \cong R / \mathfrak{p}_{2}$ for some prime $w$-ideal $\mathfrak{p}_{2}$ of $R$. Set $N_{2}:=\left(N_{1}\right)_{(w, M)}+$ $R x_{2}$. If $\left(N_{2}\right)_{(w, M)}=M$, then we have $0=N_{0} \subset\left(N_{1}\right)_{(w, M)} \subset\left(N_{2}\right)_{(w, M)}=M$, as desired. If $\left(N_{2}\right)_{(w, M)} \neq M$, then ... Continuing this process, Theorem 2.9 gives an ascending chain as in the statement of the theorem.

It was shown in $[7$, Corollary 3.3] that for a $w$-module $M$ over a domain $R$, $M$ is injective if and only if $\operatorname{Ext}_{R}^{1}(R / I, M)=0$ for any $w$-ideal $I$ of $R$. This result readily extends to any commutative ring by using [16, Theorem 3.6], and can be strengthened as follows.

Theorem 2.7'. Let $R$ be a $w$-Noetherian ring and let $E$ be a $w$-module. Then $E$ is injective if (and only if) $\operatorname{Ext}_{R}^{1}(R / \mathfrak{p}, E)=0$ for any prime $w$-ideal $\mathfrak{p}$ of $R$.

Proof. Let $M$ be a GV-torsion-free finitely generated $R$-module. By Theorem 2.10 , there is a finite ascending chain of relative $w$-submodules of $M$ :

$$
0=N_{0} \subset\left(N_{1}\right)_{(w, M)} \subset\left(N_{2}\right)_{(w, M)} \subset \cdots \subset\left(N_{n-1}\right)_{(w, M)} \subset\left(N_{n}\right)_{(w, M)}=M
$$

such that $\left(N_{i-1}\right)_{(w, M)} \subset N_{i}$ and $N_{i} /\left(N_{i-1}\right)_{(w, M)} \cong R / \mathfrak{p}_{i}$ for some prime $w$-ideal $\mathfrak{p}_{i}$ of $R, i=1, \ldots, n$. Thus we have $\operatorname{Ext}_{R}^{1}\left(N_{1}, E\right)=0$. Set $M_{i}:=$ $\left(N_{i}\right)_{(w, M)}$. Since $0 \rightarrow N_{1} \rightarrow M_{1} \rightarrow M_{1} / N_{1} \rightarrow 0$ is exact and $M_{1} / N_{1}$ is GVtorsion, we have $\operatorname{Ext}_{R}^{1}\left(M_{1}, E\right)=0$. Since $0 \rightarrow M_{1} \rightarrow N_{2} \rightarrow N_{2} / M_{1} \rightarrow 0$ is exact and $N_{2} / M_{1} \cong R / \mathfrak{p}_{2}$, we have $\operatorname{Ext}_{R}^{1}\left(N_{2}, E\right)=0$. As $0 \rightarrow N_{2} \rightarrow M_{2} \rightarrow$ $M_{2} / N_{2} \rightarrow 0$ is exact and $M_{2} / N_{2}$ is GV-torsion, we have also $\operatorname{Ext}_{R}^{1}\left(M_{2}, E\right)=0$. 
Continuing this process, we have that $\operatorname{Ext}_{R}^{1}(M, E)=0$. Therefore $E$ is injective.

Recall from [12, Definition 1.2] that a sequence $A \rightarrow B \rightarrow C$ of $R$-modules and homomorphisms is said to be $w$-exact if the sequence $A_{\mathrm{m}} \rightarrow B_{\mathrm{m}} \rightarrow C_{\mathrm{m}}$ is exact for any maximal $w$-ideal $\mathfrak{m}$ of $R$. The following result comes from $[12$, Theorem 3.4].

Lemma 2.11. Let $A \rightarrow B \rightarrow C \rightarrow 0$ be a w-exact sequence and let $N$ be a $w$-module. Then

$$
0 \rightarrow \operatorname{Hom}_{R}(C, N) \rightarrow \operatorname{Hom}_{R}(B, N) \rightarrow \operatorname{Hom}_{R}(A, N)
$$

is exact.

Recall from [12] that a GV-torsion-free $R$-module $M$ is of finite type if and only if there exists a $w$-exact sequence $F_{0} \rightarrow M \rightarrow 0$, while a GV-torsionfree $R$-module $M$ is said to be of finitely presented type if there is an $w$-exact sequence $F_{1} \rightarrow F_{0} \rightarrow M \rightarrow 0$, where $F_{0}$ and $F_{1}$ are finitely generated free $R$-modules. A commutative $\operatorname{ring} R$ is called a $w$-coherent ring if every finite type ideal of $R$ is of finitely presented type. Then it is shown in [12, Corollary 3.3 ] that every $w$-Noetherian ring is $w$-coherent.

Lemma 2.12. Let $\mathfrak{p}$ be a prime $w$-ideal of $R$. Suppose $M$ is a finitely presented type module and $N$ is a w-module. Then we have:

(a) The natural homomorphism

$$
\theta: \operatorname{Hom}_{R}(M, N)_{\mathfrak{p}} \rightarrow \operatorname{Hom}_{R_{\mathfrak{p}}}\left(M_{\mathfrak{p}}, N_{\mathfrak{p}}\right)
$$

is an isomorphism.

(b) If $R$ is $w$-Noetherian and $M$ is finitely generated, then the induced homomorphism

$$
\bar{\theta}: \operatorname{Ext}_{R}^{1}(M, N)_{\mathfrak{p}} \rightarrow \operatorname{Ext}_{R_{\mathfrak{p}}}^{1}\left(M_{\mathfrak{p}}, N_{\mathfrak{p}}\right)
$$

is an isomorphism.

Proof. (a) Because $M$ is a finitely presented type $R$-module, there exist finitely generated free $R$-modules $F_{0}$ and $F_{1}$ such that $F_{1} \rightarrow F_{0} \rightarrow M \rightarrow 0$ is a $w$-exact sequence. Then

$$
\left(F_{1}\right)_{\mathfrak{p}} \rightarrow\left(F_{0}\right)_{\mathfrak{p}} \rightarrow M_{\mathfrak{p}} \rightarrow 0
$$

is exact. By Lemma 2.11, we have the following commutative diagram with exact rows:

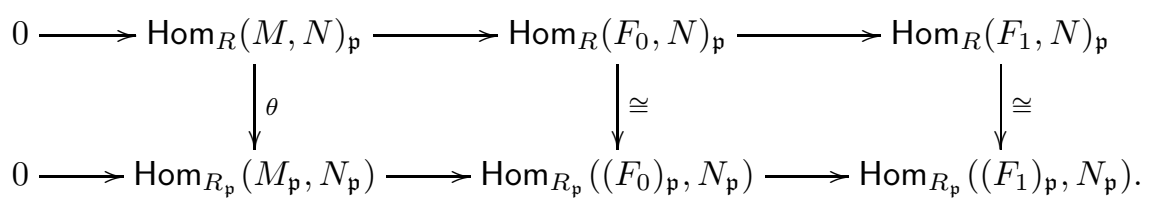

So $\theta$ is an isomorphism by the Five Lemma. From the argument above we remark that if $M$ is of finite type, then $\theta$ is a monomorphism. 
For (b), we assume first that $M$ is finitely generated. Let $0 \rightarrow A \rightarrow F \rightarrow$ $M \rightarrow 0$ be exact, where $F$ is finitely generated free. Thus $A$ is of finite type. Since $R$ is $w$-Noetherian, $A$ is of finitely presented. Then we have the following commutative diagram with exact rows:

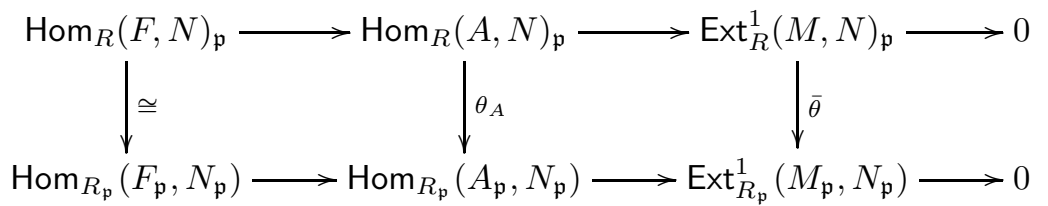

Note that $\theta_{A}$ is an isomorphism. Hence $\bar{\theta}$ is an isomorphism.

Theorem 2.13. Let $R$ be a w-Noetherian ring. If $E$ is a $G V$-torsion-free injective $R$-module, then $E_{\mathfrak{p}}$ is an injective $R_{\mathfrak{p}}$-module for any maximal $w$-ideal $\mathfrak{p}$ of $R$.

Proof. Suppose $E$ is injective over $R$. Let $X$ be a finitely generated $R_{\mathfrak{p}}$-module. Then we can write $X=M_{\mathfrak{p}}$ for some finitely generated $R$-module $M$. Since $R$ is $w$-Noetherian, $M$ is of finitely presented type. By Lemma 2.12, we have that

$$
\operatorname{Ext}_{R_{\mathfrak{p}}}^{1}\left(X, E_{\mathfrak{p}}\right)=\operatorname{Ext}_{R_{\mathfrak{p}}}^{1}\left(M_{\mathfrak{p}}, E_{\mathfrak{p}}\right)=\operatorname{Ext}_{R}^{1}(M, E)_{\mathfrak{p}}=0 .
$$

Hence $E_{\mathfrak{p}}$ is injective over $R_{\mathfrak{p}}$.

In the previous version of this paper it is shown that each localization of a GV-torsion-free injective module over a coherent SM domain is injective by using the same arguments as those of [2, Lemma 9 and Theorem 10]. As the referee suggests, Theorem 2.13 and the result just mentioned above can be better improved by proving that each localization of any GV-torsion-free injective $R$-module is injective if $R$ is $w$-Noetherian. First it is necessary to show the following result, which is the $w$-theoretic analogue of the Bass-MatlisPapp Theorem for Noetherian rings (cf., [9]):

Theorem 2.14. The following conditions are equivalent for a commutative $\operatorname{ring} R$ :

(1) $R$ is a $w$-Noetherian ring;

(2) each direct sum of $G V$-torsion-free injective $R$-modules is injective;

(3) each $G V$-torsion-free injective $R$-module is a direct sum of indecomposable $G V$-torsion-free injective $R$-modules.

Proof. (1) $\Leftrightarrow(2)[15$, Theorem 4.4] (or [8, Theorem 2.9]).

$(1) \Rightarrow(3)[15$, Theorem 4.5(4)] (or [8, Corollary 2.8]).

$(3) \Rightarrow(2)$ This follows by an easy modification of the proof of $[11$, Theorem $2]$. However, for the sake of completeness we give its proof here. Let $\left\{E_{i}\right\}_{i \in I}$ be a family of $\mathrm{GV}$-torsion-free injective $R$-modules, where $I$ is an index set and let $F:=E\left(\bigoplus_{i \in I} E_{i}\right)$. Then by hypothesis, $F=\bigoplus_{j \in J} F_{j}$, where each $F_{j}$ is an indecomposable $\mathrm{GV}$-torsion-free injective $R$-module and $J$ is an index set. 
Since $\bigoplus_{i \in I} E_{i}$ is an essential extension of $F$, it has a nonzero intersection with each $F_{j}$. Hence $B_{j}:=F_{j} \cap\left(E_{i_{1}} \oplus \cdots \oplus E_{i_{n}}\right) \neq 0$ for some $i_{1}, \ldots, i_{n}$ (depending on $j$ ). But $F_{j}$ is indecomposable, so $F_{j}=E\left(B_{j}\right) \subseteq E_{i_{1}} \oplus \cdots \oplus E_{i_{n}}$. It follows that $\bigoplus_{i \in I} E_{i}=F$ is injective.

There is an example of a non-Noetherian (coherent) SM domain: Let $K$ be a field and $\left\{X_{\alpha}\right\}$ be a countably infinite set of indeterminates. Then the polynomial ring $R:=K\left[\left\{X_{\alpha}\right\}\right]$ is a (coherent) SM domain which is not Noetherian. It follows from [3, Corollary 17] that the localization $E_{S}$ of any injective $R$-module $E$ is an injective $R_{S}$-module. Thus the following result is a generalization of the Noetherian case, and shows that one aspect of the previous example carries over to the most general case.

Theorem 2.15. Let $R$ be a $w$-Noetherian ring. Then each localization of any $G V$-torsion-free injective $R$-module is injective.

Proof. Let $E^{\prime}$ be an injective $w$-module over $R$ and $S$ a multiplicative subset of $R$. Since $R_{S}$ is flat over $R$, a module over $R_{S}$ is injective if and only if it is injective over $R$. So, since $E^{\prime}$ is a direct sum of indecomposable modules of the form $E(R / \mathfrak{p})$ where $\mathfrak{p}$ is a prime $w$-ideal (by Theorem 2.14 and [15, Theorem $4.5(1)])$, it is enough to show that $E_{S}$ is injective over $R$ if $E:=E(R / \mathfrak{p})$. Since $E$ is a module over $R_{\mathfrak{p}}$, if $S^{\prime}$ is the image of $S$ by the natural map $R \rightarrow R_{\mathfrak{p}}$, then $E_{S} \cong E_{S^{\prime}}$. Since $R_{\mathfrak{p}}$ is Noetherian, $E_{S^{\prime}}$ is injective over $R_{\mathfrak{p}}$, whence $E_{S}$ is injective over $R$.

Acknowledgement. The authors would like to thank the referee for his/her very insightful suggestions which resulted in an improved version of the paper.

\section{References}

[1] F. Couchot, Localization of injective modules over valuations rings, Proc. Amer. Math. Soc. 134 (2006), no. 4, 1013-1017.

[2] Localization of injective modules over arithmetical rings, Comm. Algebra 37 (2009), no. 10, 3418-3423.

[3] E. C. Dade, Localization of injective modules, J. Algebra 69 (1981), no. 2, 416-425.

[4] L. Fuchs and L. Salce, Modules over Non-Noetherian Domains, Mathematical Surveys and Monographs 84, AMS, Providence, RI, 2001.

[5] R. Gilmer, Multiplicative Ideal Theory, Queen's Papers in Pure and Applied Mathematics 90, Queen's University, Kingston, Ontario, 1992.

[6] B. G. Kang, On the converse of a well-known fact about Krull domains, J. Algebra 124 (1989), no. 2, 284-299.

[7] H. Kim, Module-theoretic characterizations of t-linkative domains, Comm. Algebra 36 (2008), no. 5, 1649-1670.

[8] H. Kim, E. S. Kim, and Y. S. Park, Injective modules over strong Mori domains, Houston J. Math. 34 (2008), no. 2, 349-360.

[9] E. Matlis, Injective modules over Noetherian rings, Pacific J. Math. 8 (1958), no. 3, $511-528$.

[10] J. J. Rotman, An Introduction to Homological Algebra, 2nd ed., Springer, New York, 2009. 
[11] B. Stenström, Direct sum decompositions in Grothendiek categories, Ark. Mat. 7 (1968), no. 5, 427-432.

[12] F. Wang, Finitely presented type modules and w-coherent rings, J. Sichuan Normal Univ. 33 (2010), no. 1, 1-9.

[13] F. Wang and R. L. McCasland, On w-modules over strong Mori domains, Comm. Algebra 25 (1997), no. 4, 1285-1306.

[14] - On strong Mori domains, J. Pure Appl. Algebra 135 (1999), no. 2, 155-165.

[15] F. Wang and J. Zhang, Injectve modules over $w$-Noetherian rings, Acta Math. Sinica (Chin. Ser.) $5 \mathbf{3}$ (2010), no. 6, 1119-1130.

[16] H. Yin, F. Wang, X. Zhu, and Y. Chen, w-modules over commutative rings, J. Korean Math. Soc. 48 (2011), no. 1, 207-222.

HWANKOO KIM

Department of InFormation SECURITY

HOSEO UNIVERSITY

ASAN 336-795, KOREA

E-mail address: hkkim@hoseo.edu

FANGGUI WANG

Institute of Mathematics and Software Science

Sichuan Normal University

Chengdu 610068, P. R. China

E-mail address: wangfg2004@163.com 